Nutrition Science en évolution

La revue de l'Ordre professionnel des diététistes du Québec

\title{
Congrès de La Société américaine de nutrition
}

\section{Véronique Gingras}

Volume 16, numéro 2, automne 2018

URI : https://id.erudit.org/iderudit/1054347ar

DOI : https://doi.org/10.7202/1054347ar

Aller au sommaire du numéro

Éditeur(s)

Ordre professionnel des diététistes du Québec

ISSN

2561-620X (numérique)

Découvrir la revue

Citer ce document

Gingras, V. (2018). Congrès de La Société américaine de nutrition. Nutrition

Science en évolution, 16(2), 41-43. https://doi.org/10.7202/1054347ar d'utilisation que vous pouvez consulter en ligne.

https://apropos.erudit.org/fr/usagers/politique-dutilisation/ 


\section{CONGRĖS DE LA SOCIÉTÉ AMÉRICAINE DE NUTRITION}

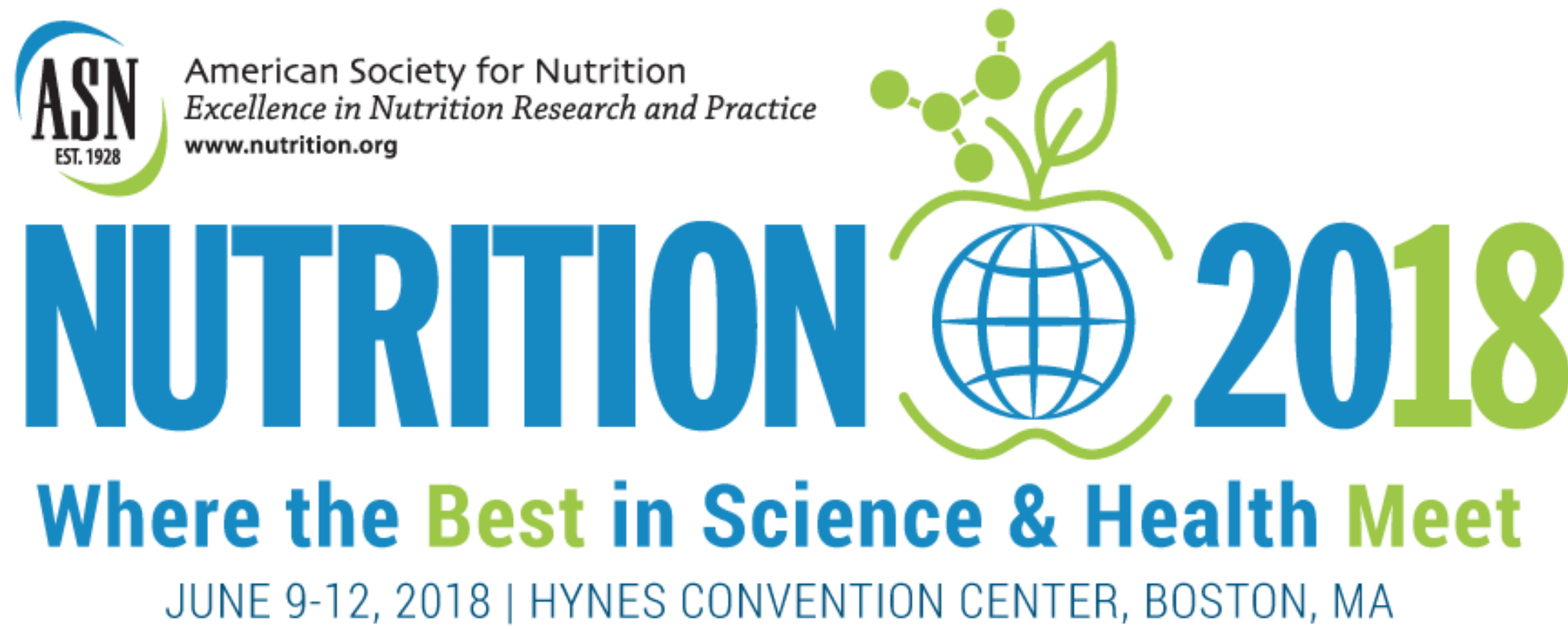

Véronique Gingras, Dt.P., Ph. D. Stagiaire postdoctorale, Department of Population Medicine Harvard Medical School - Harvard Pilgrim Health Care Institute

La Société américaine de nutrition a tenu sa première réunion scientifique indépendante pour faire part des nouveautés scientifiques dans le domaine de la nutrition clinique, fondamentale, populationnelle et translationnelle. Environ 3000 participants de plus de 1000 organisations provenant de 69 pays y ont participé. Voici le résumé de trois séances présentées dans le cadre de cette réunion.

\section{COMPRENDRE LES INTERRELATIONS ENTRE LES CHANGEMENTS CLIMATIQUES ET ENVIRONNEMENTAUX, LA SANTÉ, L'AGRICULTURE ET L'AMÉLIORATION NUTRITIONNELLE — UNE ÉTUDE DE CAS: LE DIABÈTE}

Lindsay M. Jaacks, Ph. D. Harvard T.H. Chan School of Public Health

Donald R. Ort, Ph. D.

Université d'lllinois

Christian J. Peters, Ph. D.

Université Tufts

Comprendre les liens et les défis du continuum entre l'approvisionnement alimentaire, la nutrition et la santé est essentiel pour développer des systèmes et des politiques efficaces. Lindsay Jaacks a d'abord discuté des associations positives ob- servées entre le diabète et la consommation de viande, entre le diabète et l'exposition à certains pesticides, et de l'association négative observée entre le diabète et la consommation de fruits et légumes. Elle a rappelé que la malnutrition demeure une problématique majeure. Des systèmes alimentaires inadéquats contribuent autant à cette problématique qu’à une surconsommation ou une consommation sous-optimale de certains aliments menant à un risque accru de diabète. Donald Ort a ensuite discuté de l'association entre les changements climatiques et les aliments. Les produits céréaliers sont à cet égard les aliments les mieux étudiés. Il a été démontré qu'une augmentation de la concentration ambiante en dioxyde de carbone (CO2) est associée à une diminution du contenu des grains entiers en protéines, en zinc et en fer. Deux mécanismes expliquent 
42

cette association : 1) la stimulation accrue de la photosynthèse, causant une croissance accélérée et une dilution des nutriments ; 2) une réduction de l'évaporation par la plante, réduisant ainsi le flot de nutriments et de minéraux provenant du sol. Finalement, Christian Peters a discuté des capacités du système alimentaire actuel à rejoindre les recommandations nutritionnelles pour réduire le risque de diabète, en citant l'exemple de la consommation de fruits et légumes. D'abord, il a souligné l'écart majeur entre la consommation actuelle de certains groupes d'aliments (p. ex. légumes verts et légumineuses) et les recommandations nutritionnelles. Pour l'atteinte des recommandations à l'échelle de la population, plusieurs défis liés à la production alimentaire persistent, comme l'émission de gaz à effets de serre, l'utilisation d'eau et d'énergie et l'espace disponible. Pour favoriser une saine alimentation, l'adaptation de notre système actuel d'agriculture s'impose.

\section{UNE CALORIE EST-ELLE VRAIMENT UNE CALORIE? REFORMULONS LA QUESTION}

James 0. Hill, Ph. D.

University of Colorado School of Medicine

Kevin D. Hall, Ph. D.

National Institute of Diabetes and

Digestive and Kidney Diseases/

National Institutes of Health

Eric Ravussin, Ph. D.

Pennington Biomedical Research

Center

Frank B. Hu, M. D., Ph. D.

Harvard T.H. Chan School of Public Health

John M. Jakicic, Ph. D.

Université de Pittsburgh

James Hill a ouvert la séance en précisant que l'équilibre énergétique (EE) n'est pas un concept simple; chaque facteur affectant le poids corporel le fait par la régulation métabolique de l'EE. L'obésité serait donc une adaptation à notre environnement. Les facteurs qui influencent l'EE étant nombreux, on obtient un système complexe et adaptatif. M. Hall a poursuivi en discutant des effets sur la perte de poids des diètes faibles en glucides ou lipides, indépendamment d'une réduction calorique. À son avis, les diètes faibles en glucides sont aussi efficaces que les autres types de diètes sur la perte de poids puisque les effets sur la dépense énergétique et l'adiposité semblent dépendre très peu de la proportion de glucides versus celle des lipides dans la diète. Eric Ravussin a quant à lui discuté de l'effet de la flexibilité métabolique dans les cas d'obésité et de diabète de type 2. La flexibilité métabolique est la capacité du métabolisme à s'adapter efficacement par l'absorption, le transport, le stockage et l'utilisation des substrats selon la disponibilité et les demandes en énergie. Elle est difficile à évaluer, mais la détermination des mécanismes sous-jacents de la non-flexibilité métabolique pourrait mener à de nouvelles avenues thérapeutiques pour améliorer la santé métabolique. Dr Hu a poursuivi en parlant de l'importance de considérer les schémas alimentaires. Il a montré que la qualité nutritionnelle globale peut influencer le risque de maladies chroniques indépendamment de l'obésité. Selon $\mathrm{Dr} \mathrm{Hu}$, la qualité nutritionnelle ne doit pas considérer que les macronutriments, mais aussi la transformation des aliments et l'ajout de certains ingrédients ( $\mathrm{p}$. ex. sodium et sucres). Selon lui, une calorie est une calorie, mais sa provenance joue un rôle essentiel. Finalement, M. Jakicic a conclu en discutant du rôle de l'activité physique dans la dynamique de l'EE. Il rapporte que certaines études ont suggéré que la diète seule ou la combinaison de la diète et de l'activité physique produisent des résultats similaires sur la perte de poids en raison d'une com- pensation de la dépense énergétique liée à la pratique d'activité physique (c.-à-d. que les participants tendent à diminuer leur dépense énergétique pour le reste de la journée à la suite d'une séance d'activité physique). Au dire de M. Jakicic, s’intéresser aux schémas de pratique d'activité physique et non seulement à la dépense énergétique totale constitue une avenue prometteuse.

\section{LA PRÉVENTION DES ALLERGIES ALIMENTAIRES ET DES MALADIES ATOPIQUES: LA PROGRESSION DE L'ATOPIE PEUT-ELLE ÊTRE STOPPÉE?}

Susan Prescott, M. D., Ph. D. University of Western Australia

David M. Fleischer, M. D. University of Colorado School of Medicine

Robert Boyle, M. D., Ph. D. Imperial College London

Carina Venter, Ph. D., RD

University of Colorado School

of Medicine

Marion Groetch, MS, RDN Icahn School of Medicine at Mount Sinai

Dre Prescott a mentionné que les changements nutritionnels complexes survenus au cours des derniers siècles sont probablement mis en cause dans l'épidémie d'allergies chez les nourrissons et les enfants, bien que cette relation demeure incomprise. Facteur déterminant du microbiome, l'alimentation influence également la santé immunitaire et métabolique. La mise en place de stratégies nutritionnelles et écologiques pour contrer un déséquilibre immunitaire est donc essentielle. Dr Fleischer a pour sa part discuté de l'introduction des aliments allergènes chez les nourrissons, plus particulièrement de l'arachide. L'étude Learning Early About Peanut Allergy a montré que l'introduction précoce des arachides avait un effet 
bénéfique sur la réduction de l'allergie à cet aliment chez les enfants à risque. Selon l'étude, l'introduction des arachides serait optimale entre 6 et 9 mois de vie. Toutefois, selon de récentes études, les parents demeurent hésitants à introduire en bas âge cet aliment. Aussi, il conviendrait de revoir les lignes directrices. Dr Boyle a mentionné que plusieurs lignes directrices internationales recommandent les préparations pour nourrissons hydrolysées pour la prévention des allergies chez les enfants ne pouvant être allaités. Or, ces recommandations sont basées sur une méta-analyse datant de plusieurs années qui incluait deux études depuis reconnues comme frauduleuses. Selon les nouvelles méta-analyses et revues systématiques, rien ne prouve que ces préparations préviennent les allergies au lait de vache ou l'eczéma.
Finalement, Mmes Venter et Groetch ont pesé le pour et le contre de l'introduction précoce de l'arachide. Mme Venter a discuté de la complexité d'appliquer ces recommandations et de cibler la bonne population «à risque ». Elle a également fait part de l'incertitude qui entoure la quantité requise (en grammes par semaine) pour réduire le risque d'allergie. Elle a également insisté sur l'importance de considérer les changements apportés à la composition de la diète du nourrisson (c.-à-d. la teneur en protéines, en sodium, etc.). Selon Mme Groetch, l'introduction des arachides, non seulement n'influencerait aucunement l'allaitement maternel, mais pourrait avoir un effet nutritionnel intéressant en augmentant le ratio de protéines d'origine végétale de l'alimentation.
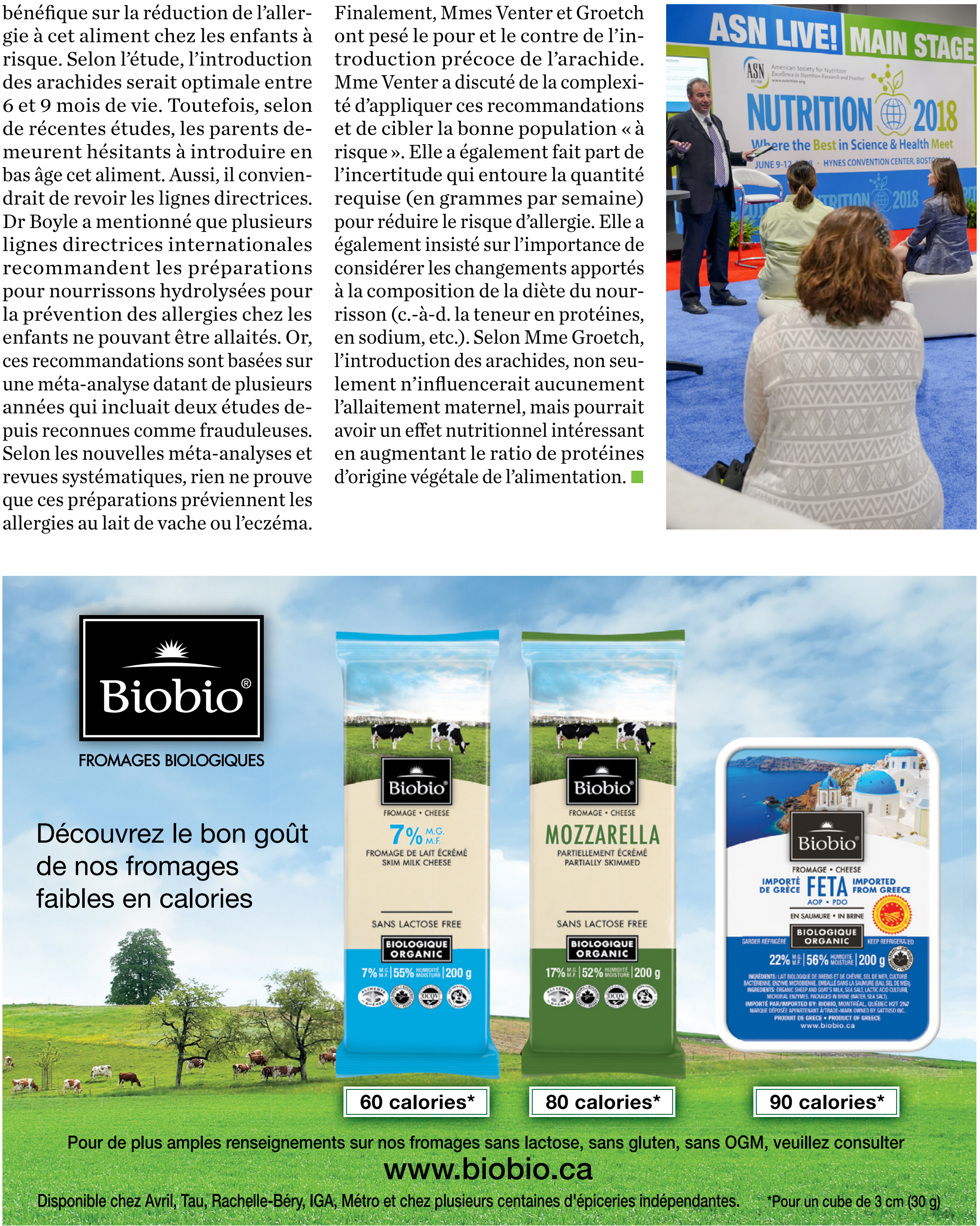